\title{
RESEARCH
}

\section{Operations on stable moduli spaces}

\author{
Søren Galatius ${ }^{1 *}$ and Oscar Randal-Williams ${ }^{2}$
}

${ }^{*}$ Correspondence:
galatius@math.ku.dk
${ }^{1}$ Department of Mathematics,
University of Copenhagen,
Copenhagen, Denmark
Full list of author information is
available at the end of the article

available at the end of the article

\begin{abstract}
We construct certain operations on stable moduli spaces and use them to compare cohomology of moduli spaces of closed manifolds with tangential structure. We obtain isomorphisms in a stable range provided the $p$-adic valuation of the Euler characteristics agree, for all primes $p$ not invertible in the coefficients for cohomology.

Keywords: Moduli spaces, Diffeomorphism groups, Homological stability, Characteristic classes

Mathematics Subject Classification: 55P47, 55R40, 57S05, 57R15, 57R90
\end{abstract}

\section{Introduction}

An influential theorem of Harer [9] shows that the cohomology of the moduli stack $\mathcal{M}_{g}$ of genus $g$ Riemann surfaces is independent of $g$ in a range of degrees called the stable range, even though there is no direct map between the moduli spaces for different genera. With rational coefficients, the cohomology in the stable range is a polynomial ring, but with more general coefficients it is best described via infinite loop spaces, as shown by [1113]. In earlier papers ([5-7], see also [8] for a survey), we have studied moduli spaces of higher-dimensional manifolds and, in some cases, have again shown that different moduli spaces have isomorphic cohomology in a range of degrees. For $n>1$, one can in most cases not make an integral comparison of moduli spaces of manifolds related by connected sum with copies of $S^{n} \times S^{n}$, at least not by an obvious generalization of the $n=1$ case, where a zig-zag of integral homology equivalences can be defined using manifolds with boundary. In this paper, we show that a comparison is possible after all, although not with all coefficient modules. We also give examples showing that assumptions on the coefficients are necessary.

\subsection{Comparing moduli spaces of closed manifolds}

All manifolds in this paper will be smooth, compact, connected, and without boundary. If $W$ denotes such a manifold, then there is a moduli space $\mathcal{M}(W)$ classifying smooth fiber bundles whose fibers are diffeomorphic to $W$. As a model, we may take $\mathcal{M}(W)=$ $B \operatorname{Diff}(W)$, the classifying space of the diffeomorphism group $\operatorname{Diff}(W)$ of $W$, equipped with the $C^{\infty}$ topology. Then for $A$ an abelian group, $H^{i}(\mathcal{M}(W) ; A)$ is the group of $H^{i}(-; A)$ valued characteristic classes of such fiber bundles.

(c) The Author(s) 2020. This article is licensed under a Creative Commons Attribution 4.0 International License, which permits use, sharing, adaptation, distribution and reproduction in any medium or format, as long as you give appropriate credit to the original author(s) and the source, provide a link to the Creative Commons licence, and indicate if changes were made. The images or other third party material in this article are included in the article's Creative Commons licence, unless indicated otherwise in a credit line to the material. If material is not included in the article's Creative Commons licence and your intended use is not permitted by statutory regulation or exceeds the permitted use, you will need to obtain permission directly from the copyright holder. To view a copy of this licence, visit http://creativecommons.org/licenses/by/4.0/. 
Now let $d=2 n$ and $W$ be a $d$-manifold. The connected sum $W \#\left(S^{n} \times S^{n}\right)$ is then well defined up to (non-canonical) diffeomorphism, as $S^{n} \times S^{n}$ admits an orientationreversing diffeomorphism, and we write $W \# g\left(S^{n} \times S^{n}\right)$ for the $g$-fold iteration of this operation. Two manifolds $W$ and $W^{\prime}$ are called stably diffeomorphic if $W \# g\left(S^{n} \times S^{n}\right)$ is diffeomorphic to $W^{\prime} \# g^{\prime}\left(S^{n} \times S^{n}\right)$ for some $g, g^{\prime} \in \mathbb{N}$. For example, any two orientable connected surfaces are stably diffeomorphic, while two non-orientable connected surfaces are stably diffeomorphic if and only if their Euler characteristic have the same parity.

In this paper, we shall ask about the relationship between $H^{*}(\mathcal{M}(W) ; A)$ and $H^{*}\left(\mathcal{M}\left(W^{\prime}\right) ; A\right)$ when $W$ and $W^{\prime}$ are stably diffeomorphic. As a special case, our main result will provide a canonical isomorphism

$$
H^{i}\left(\mathcal{M}(W) ; \mathbb{Z}_{(p)}\right) \cong H^{i}\left(\mathcal{M}\left(W^{\prime}\right) ; \mathbb{Z}_{(p)}\right)
$$

as long as these manifolds are simply-connected and of dimension $2 n>4$, and both $(-1)^{n} \chi(W)$ and $(-1)^{n} \chi\left(W^{\prime}\right)$ are large compared with $i$ and have the same $p$-adic valuation.

The precise statement of our main result applies more generally, and before giving it we first explain its natural setting. If $W$ is given an orientation $\lambda$, then there is a corresponding moduli space $\mathcal{M}^{\text {or }}(W, \lambda)$ classifying smooth fiber bundles with oriented fibers which are oriented diffeomorphic to $(W, \lambda)$, and a forgetful map $\mathcal{M}^{\text {or }}(W, \lambda) \rightarrow \mathcal{M}(W)$. Then the connected sum $W \# g\left(S^{n} \times S^{n}\right)$ inherits an orientation, well defined up to oriented diffeomorphism, and we say that $(W, \lambda)$ is oriented stably diffeomorphic to $\left(W^{\prime}, \lambda^{\prime}\right)$ provided $W \# g\left(S^{n} \times S^{n}\right)$ is oriented diffeomorphic to $W^{\prime} \# g^{\prime}\left(S^{n} \times S^{n}\right)$ for some $g, g^{\prime} \in \mathbb{N}$. In this situation, our result will also imply a canonical isomorphism $H^{i}\left(\mathcal{M}^{\text {or }}(W, \lambda) ; \mathbb{Z}_{(p)}\right) \cong$ $H^{i}\left(\mathcal{M}^{\text {or }}\left(W^{\prime}, \lambda^{\prime}\right) ; \mathbb{Z}_{(p)}\right)$, under the same hypotheses.

More generally, for a space $\Lambda$ equipped with a continuous action of $\mathrm{GL}_{d+1}(\mathbb{R})$, a $\Lambda$ structure on a $d$-manifold $W$ is a $\mathrm{GL}_{d}(\mathbb{R})$-equivariant map $\lambda: \operatorname{Fr}(T W) \rightarrow \Lambda$, or, equivalently, a $\mathrm{GL}_{d+1}(\mathbb{R})$-equivariant map $\operatorname{Fr}\left(\varepsilon^{1} \oplus T W\right) \rightarrow \Lambda$. For example, if $\Lambda=\{ \pm 1\}$ on which $\mathrm{GL}_{d+1}(\mathbb{R})$ acts by multiplication by the sign of the determinant, then a $\Lambda$-structure $\lambda: \operatorname{Fr}(T W) \rightarrow\{ \pm 1\}$ is the same thing as an orientation: It distinguishes oriented frames from non-oriented ones. Two $\Lambda$-structures on the same manifold are homotopic if they are homotopic through equivariant maps, and $(W, \lambda)$ is $\Lambda$-diffeomorphic to $\left(W^{\prime}, \lambda^{\prime}\right)$ if there exists a diffeomorphism $\phi: W \rightarrow W^{\prime}$ such that $\lambda \circ D \phi$ is homotopic to $\lambda^{\prime}$. The usual embedding of $S^{n} \times S^{n} \subset \mathbb{R}^{2 n+1}$ as the boundary of a thickened $S^{n} \times\{0\} \subset \mathbb{R}^{n+1} \times \mathbb{R}^{n}$ gives a trivialization of $\varepsilon^{1} \oplus T\left(S^{n} \times S^{n}\right)$ and a $\Lambda$-structure on $W$ extends to one on $W \#\left(S^{n} \times S^{n}\right)$, canonically up to $\Lambda$-diffeomorphism. For two pairs $(W, \lambda)$ and $\left(W^{\prime}, \lambda^{\prime}\right)$ consisting of a manifold and a $\Lambda$-structure, we say that they are stably $\Lambda$-diffeomorphic if $W \# g\left(S^{n} \times S^{n}\right)$ is $\Lambda$-diffeomorphic to $W^{\prime} \# g^{\prime}\left(S^{n} \times S^{n}\right)$ for some $g, g^{\prime} \in \mathbb{N}$.

There is a moduli space $\mathcal{M}^{\Lambda}(W, \lambda)$ parametrizing smooth fiber bundles $\pi: E \rightarrow X$ with $d$-dimensional fibers, and where the fiberwise tangent bundle $T_{\pi} E$ is equipped with an equivariant map $\operatorname{Fr}\left(\varepsilon^{1} \oplus T_{\pi} E\right) \rightarrow \Lambda$, such that all fibers of $\pi$ are $\Lambda$-diffeomorphic to $(W, \lambda)$. Our main result is then as follows.

Theorem 1.1 Let $\Lambda$ be as above, and let $\lambda$ and $\lambda^{\prime}$ be $\Lambda$-structures on $W$ and $W^{\prime}$ such that $(W, \lambda)$ is stably $\Lambda$-diffeomorphic to $\left(W^{\prime}, \lambda^{\prime}\right)$. For an abelian group $A$, there is a canonical isomorphism

$$
H^{i}\left(\mathcal{M}^{\Lambda}(W, \lambda) ; A\right) \cong H^{i}\left(\mathcal{M}^{\Lambda}\left(W^{\prime}, \lambda^{\prime}\right) ; A\right)
$$


induced by a zig-zag of maps of spaces, provided

(i) $d=2 n>4$ and $W$ and $W^{\prime}$ are simply connected,

(ii) the integers $(-1)^{n} \chi(W)$ and $(-1)^{n} \chi\left(W^{\prime}\right)$ are both $\geq 4 i+C$, where

$$
C=6+\min \left\{(-1)^{n} \chi\left(W_{0}\right) \mid\left(W_{0}, \lambda_{0}\right) \text { stably } \Lambda \text {-diffeomorphic to }(W, \lambda) \text { and }\left(W^{\prime}, \lambda^{\prime}\right)\right\} .
$$

(iii) $\chi(W)$ and $\chi\left(W^{\prime}\right)$ are both non-zero, and $v_{p}(\chi(W))=v_{p}\left(\chi\left(W^{\prime}\right)\right)$ for all primes $p$ which are not invertible in $\operatorname{End}_{\mathbb{Z}}(A)$.

In Sect. 4, we give an example showing the third condition cannot be relaxed. The main results of [5-7], summarized in [8], provide a map

$$
\mathcal{M}^{\Lambda}(W, \lambda) \longrightarrow\left(\Omega^{\infty} M T \Theta\right) / / \operatorname{hAut}(u)
$$

which induces an isomorphism on homology in a range of degrees, when regarded as a map to the path component which it hits. Similarly there is a map

$$
\mathcal{M}^{\Lambda}\left(W^{\prime}, \lambda^{\prime}\right) \longrightarrow\left(\Omega^{\infty} M T \Theta\right) / / \operatorname{hAut}(u)
$$

which induces an isomorphism on homology in a range of degrees, when regarded as a map to the path component which it hits. The definition of the codomains is recalled below. However, if $\chi(W) \neq \chi\left(W^{\prime}\right)$, then these two maps land in different path components, and the problem becomes to compare the homology of these two path components.

Remark 1.2 Using the results of Friedrich [4], Theorem 1.1 can be extended to manifolds with virtually polycyclic fundamental groups. In this case, the constant $C$ should be replaced by $C+4+2 h$ where $h$ denotes the Hirsch length of the common fundamental group of $W$ and $W^{\prime}$.

\subsection{Operations on infinite loop spaces}

The data involved in defining the common target of the maps (1.1) and (1.2) is a $\mathrm{GL}_{2 n}(\mathbb{R})$ equivariant fibration $u: \Theta \rightarrow \Lambda$ with domain which is cofibrant as a $\mathrm{GL}_{2 n}(\mathbb{R})$-space. Letting $B$ denote the Borel construction $\Theta / / \mathrm{GL}_{2 n}(\mathbb{R}), M T \Theta$ is then the Thom spectrum of the inverse of the canonical $2 n$-dimensional vector bundle over $B$, and $\Omega^{\infty} M T \Theta$ is its associated infinite loop space. By functoriality, the group-like topological monoid hAut $(\Theta)$ of $\mathrm{GL}_{2 n}(\mathbb{R})$-equivariant homotopy equivalences $f: \Theta \rightarrow \Theta$ acts on the infinite loop space $\Omega^{\infty} M T \Theta$, so the group-like submonoid hAut $(u)=\{f \in \operatorname{hAut}(\Theta) \mid u \circ f=u\}$ does too. The target

$$
\left(\Omega^{\infty} M T \Theta\right) / / \operatorname{hAut}(u)
$$

of the maps (1.1) and (1.2) is the Borel construction for this action.

In order to prove Theorem 1.1, we shall construct certain operations on the space $\Omega^{\infty} M T \Theta$, in the case where the $\mathrm{GL}_{2 n}(\mathbb{R})$-space $\Theta$ is obtained by restriction from a cofibrant $\mathrm{GL}_{2 n+1}(\mathbb{R})$-space $\bar{\Theta}$. The space $\bar{B}=\bar{\Theta} / / \mathrm{GL}_{2 n+1}(\mathbb{R})$ carries a canonical $(2 n+1)$ dimensional vector bundle, and $M T \bar{\Theta}$ denotes its associated Thom spectrum; as above, by functoriality, it carries an action of the monoid hAut $(\bar{\Theta})$ of $\mathrm{GL}_{2 n+1}(\mathbb{R})$-equivariant homotopy equivalences $f: \bar{\Theta} \rightarrow \bar{\Theta}$. 
A key construction in this paper is a homotopy pullback diagram of infinite loop spaces, equivariant for hAut $(\bar{\Theta})$, of the form

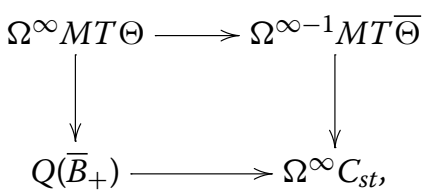

whose bottom right corner has $\pi_{0} \cong \mathbb{Z} / 2$ and all higher homotopy groups are 2-power torsion, and the bottom horizontal map induces a surjection on $\pi_{1}$. It induces an isomorphism

$$
\pi_{0} M T \Theta \stackrel{\cong}{\rightarrow}\left\{(\chi, x) \in \mathbb{Z} \times \pi_{-1} M T \bar{\Theta} \mid \chi \bmod 2=w_{2 n}(x)\right\}
$$

whose first coordinate is given by the Euler class and whose second coordinate is given by the stabilization map. To explain this claim and its notation, first note that the $2 n$ dimensional vector bundle over $B$ has an Euler class $e \in H^{2 n}\left(B ; \mathbb{Z}^{w_{1}}\right)$, where the coefficients are twisted by the determinant of this vector bundle, and under the Thom isomorphism this gives a class $e \smile u_{-2 n} \in H^{0}(M T \Theta ; \mathbb{Z})$. Then $\chi$ is the value of this spectrum cohomology class on the Hurewicz image of an element of $\pi_{0} M T \Theta$; geometrically, it assigns to such an element the Euler characteristic of a manifold representing it. Similarly, the $(2 n+1)$ dimensional vector bundle over $\bar{B}$ has a $2 n$th Stiefel-Whitney class $w_{2 n} \in H^{2 n}(\bar{B} ; \mathbb{Z} / 2)$, and under the Thom isomorphism this gives a class $w_{2 n} \smile u_{-2 n-1} \in H^{-1}(M T \bar{\Theta} ; \mathbb{Z} / 2)$. Then $w_{2 n}(x)$ denotes the value of this spectrum cohomology class on the Hurewicz image of $x$.

Theorem 1.3 For $\chi \in \mathbb{Z}$, write $\Omega_{\chi}^{\infty} M T \Theta$ for the inverse image of $\chi$ under the map $\Omega^{\infty} M T \Theta \rightarrow \mathbb{Z}$ induced by the class $e \smile u_{-2 n} \in H^{0}(M T \Theta ; \mathbb{Z})$, i.e., the union of the path components of the form $(\chi$, ?) under the bijection (1.4).

For any odd number $q$, there exists a self-map $M T \Theta \rightarrow M T \Theta$ inducing a map

$$
\psi^{q}: \Omega_{\chi}^{\infty} M T \Theta \longrightarrow \Omega_{q \chi}^{\infty} M T \Theta
$$

such that

(i) $\psi^{q}$ commutes (strictly) with the action of hAut $(\bar{\Theta})$,

(ii) $\psi^{q}$ is over the identity map of $\Omega^{\infty-1} M T \bar{\Theta}$,

(iii) $\psi^{q}$ induces an isomorphism in homology with coefficients in any $\mathbb{Z}\left[q^{-1}\right]$-module.

We shall also prove a version of Theorem 1.3 for $q=2$, although it will be marginally weaker in that rather than the map $\psi^{q}$ being defined integrally and inducing an isomorphism with coefficients in any $\mathbb{Z}\left[q^{-1}\right]$-module, the map $\psi^{2}$ will only be defined after localizing the spaces involved away from 2 .

Theorem 1.4 In the setup of Theorem 1.3, if $\chi$ is even, then there is an hAut $(\bar{\Theta})$ equivariant weak equivalence of localized spaces

$$
\psi^{2}:\left(\Omega_{\chi}^{\infty} M T \Theta\right)\left[\frac{1}{2}\right] \longrightarrow\left(\Omega_{2 \chi}^{\infty} M T \Theta\right)\left[\frac{1}{2}\right]
$$

over the identity map of $\left(\Omega^{\infty-1} M T \bar{\Theta}\right)\left[\frac{1}{2}\right]$. 
The operations in Theorems 1.3 and 1.4 will arise from self-maps of the lower left corner in (1.3).

The proof of Theorem 1.1 will use these operations to give endomorphisms of the space $\left(\Omega^{\infty} M T \Theta\right) / /$ hAut $(u)$ which mix path components, allowing us to compare the path components hit by the maps (1.1) and (1.2). This strategy is analogous to arguments of Bendersky-Miller [2] and Cantero-Palmer [3] for cohomology of configuration spaces. This strategy has also been used by Krannich [10] to show that $H^{i}\left(\mathcal{M}^{\text {or }}(W, \lambda) ; A\right) \cong$ $H^{i}\left(\mathcal{M}^{\text {or }}(W \# \Sigma, \lambda) ; A\right)$ for $(W, \lambda)$ an oriented manifold of dimension $2 n>4$ and $\Sigma$ an exotic sphere, in a stable range of degrees when the order of $[\Sigma] \in \Theta_{2 n}$ is invertible in $\operatorname{End}_{\mathbb{Z}}(A)$.

\section{Proof of Theorem 1.1}

We first explain how to deduce Theorem 1.1 from Theorems 1.3 and 1.4.

Let $\lambda: \operatorname{Fr}\left(\varepsilon^{1} \oplus T W\right) \stackrel{\rho}{\rightarrow} \bar{\Theta} \stackrel{\bar{u}}{\rightarrow} \Lambda$ be a factorization into an $n$-connected $\mathrm{GL}_{2 n+1}(\mathbb{R})$ equivariant cofibration $\rho$ and a $n$-co-connected $\mathrm{GL}_{2 n+1}(\mathbb{R})$-equivariant fibration $\bar{u}$, and as above we write $\Theta$ for the underlying $\mathrm{GL}_{2 n}(\mathbb{R})$-space of $\bar{\Theta}$ and $u$ for the underlying $\mathrm{GL}_{2 n}(\mathbb{R})$-equivariant map of $\bar{u}$. There is then a map

$$
\mathcal{M}^{\Lambda}(W, \lambda) \longrightarrow\left(\Omega^{\infty} M T \Theta\right) / / \operatorname{hAut}(u),
$$

which by [6, Corollary 1.9] induces an isomorphism on $i$ th (co)homology onto the path component which it hits, as long as $i \leq \frac{\bar{g}(W, \lambda)-3}{2}$. (Note that by considering a GL $2 n+1(\mathbb{R})$ space $\Lambda$ rather than a $\mathrm{GL}_{2 n}(\mathbb{R})$-space, the tangential structure $\Theta$ is "spherical" by the discussion after [8, Definition 3.2], and so the stability range is as claimed.) Here $\bar{g}(W, \lambda)$ is the stable $\Lambda$-genus of $(W, \lambda)$, the largest $g \in \mathbb{N}$ for which there exists $h \in \mathbb{N}$ such that $W \# h\left(S^{n} \times S^{n}\right)$ is $\Lambda$-diffeomorphic to $W_{0} \#(g+h)\left(S^{n} \times S^{n}\right)$ for some $\left(W_{0}, \lambda_{0}\right)$.

Let $\left(W_{0}, \lambda_{0}\right)$ be a manifold stably $\Lambda$-diffeomorphic to $(W, \lambda)$ and minimizing the quantity $(-1)^{n} \chi\left(W_{0}\right)$. Such a manifold has stable $\Lambda$-genus zero and hence for large enough $h$ we must have that $W \# h\left(S^{n} \times S^{n}\right)$ is $\Lambda$-diffeomorphic to $W_{0} \#(h+\bar{g}(W, \lambda))\left(S^{n} \times S^{n}\right)$, so

$$
\bar{g}(W, \lambda)=(-1)^{n}\left(\chi(W)-\chi\left(W_{0}\right)\right) / 2 .
$$

It follows that (2.1) is an isomorphism on $i$ th (co)homology as long as

$$
(-1)^{n} \chi(W) \geq 4 i+\left(6+(-1)^{n} \chi\left(W_{0}\right)\right) .
$$

If $\left(W^{\prime}, \lambda^{\prime}\right)$ is stably $\Lambda$-diffeomorphic to $(W, \lambda)$, then the same analysis applies, and there is a map

$$
\mathcal{M}^{\Lambda}\left(W^{\prime}, \lambda^{\prime}\right) \longrightarrow\left(\Omega^{\infty} M T \Theta\right) / / \operatorname{hAut}(u)
$$

which induces an isomorphism on $i$ th (co)homology onto the path component which it hits, as long as

$$
(-1)^{n} \chi\left(W^{\prime}\right) \geq 4 i+\left(6+(-1)^{n} \chi\left(W_{0}\right)\right) .
$$

By assumption, we may write

$$
a \cdot \chi(W)=b \cdot \chi\left(W^{\prime}\right)
$$


for integers $a$ and $b$ all of whose prime factors are invertible in $\operatorname{End}_{\mathbb{Z}}(A)$. Furthermore, the two Euler characteristics have the same parity, as (de)stabilization changes the Euler characteristic by \pm 2 , so if either $a$ or $b$ is even then both $\chi(W)$ and $\chi\left(W^{\prime}\right)$ are even too.

By Theorems 1.3 and 1.4, writing $\psi^{x}=\psi^{x / 2^{\nu_{2}(x)}} \circ\left(\psi^{2}\right)^{v_{2}(x)}$, then (after perhaps implicitly localizing away from 2 ) there are maps

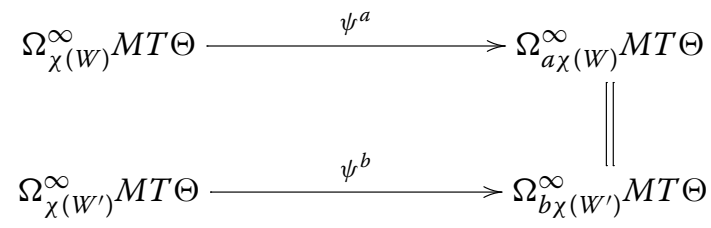

which are hAut $(\bar{\Theta})$-equivariant and induce isomorphisms on $A$-homology, as $A$ is a $\mathbb{Z}\left[a^{-1}, b^{-1}\right]$-module. By construction, these maps do not change the $\pi_{-1} M T \bar{\Theta}$ component: We now analyze the components corresponding to $W$ and $W^{\prime}$.

We now claim that $\psi^{a}([W, \rho])=\psi^{b}\left(\left[W^{\prime}, \rho^{\prime}\right]\right) \in \pi_{0}\left(\Omega^{\infty} M T \Theta\right)$ for a suitable choice of $\rho^{\prime}: \operatorname{Fr}\left(\varepsilon^{1} \oplus T W^{\prime}\right) \rightarrow \bar{\Theta}$ lifting $\lambda^{\prime}$. Since these two elements of $\pi_{0}(M T \Theta)$ have the same Euler characteristic, it suffices to arrange that they also have the same $\pi_{-1} M T \bar{\Theta}$-component. The stable $\Lambda$-diffeomorphism from $(W, \lambda)$ to $\left(W^{\prime}, \lambda^{\prime}\right)$ gives a $\Lambda$-cobordism

$$
X: W \# g\left(S^{n} \times S^{n}\right) \rightsquigarrow W^{\prime} \# g^{\prime}\left(S^{n} \times S^{n}\right),
$$

which is furthermore an $h$-cobordism. We can therefore extend the $\bar{\Theta}$-structure given by $(W, \rho)$, stabilized, to a $\bar{\Theta}$-structure on $X$ lifting the given $\Lambda$-structure, and hence obtain a $\bar{\Theta}$-manifold $\left(W^{\prime} \# g^{\prime}\left(S^{n} \times S^{n}\right), \rho^{\prime \prime}\right)$ whose underlying $\Lambda$-manifold $\left(W^{\prime} \# g^{\prime}\left(S^{n} \times S^{n}\right), u \circ \rho^{\prime \prime}\right)$ is the stabilization of $\left(W^{\prime}, \lambda^{\prime}\right)$. Now the $\bar{\Theta}$-manifolds

$$
\left(W^{\prime} \# g^{\prime}\left(S^{n} \times S^{n}\right), \rho^{\prime \prime}\right) \text { and }\left(W^{\prime}, \rho^{\prime}\right) \# g^{\prime}\left(S^{n} \times S^{n}\right)
$$

need not be $\bar{\Theta}$-diffeomorphic, but must differ by an equivalence $f: \bar{\Theta} \rightarrow \bar{\Theta}$ over $\Lambda$ (see $[6$, Lemma 9.2]). However, the $\bar{\Theta}$-structure $\rho^{\prime}$ on $W^{\prime}$ is merely a choice of lift of $\lambda^{\prime}$ along $\bar{u}$, and by rechoosing it to be $f \circ \rho^{\prime}$, we may then suppose that the manifolds (2.3) are indeed $\bar{\Theta}$-diffeomorphic. With this choice, we therefore have the desired

$$
[W, \rho]=\left[W^{\prime}, \rho^{\prime}\right] \in \pi_{-1} M T \bar{\Theta},
$$

using the $\bar{\Theta}$-cobordism $X$ and the fact that this cobordism theory is insensitive to stabilization by standard $S^{n} \times S^{n}$ s.

Denoting by $[[W, \lambda]] \subset \pi_{0} M T \Theta$ the $\pi_{0}$ hAut $(\bar{u})$-orbit of $[W, \rho]$, and similarly $\left[\left[W^{\prime}, \lambda^{\prime}\right]\right]$, and using the forgetful homomorphism hAut $(\bar{u}) \rightarrow \operatorname{hAut}(\bar{\Theta})$ to let the monoid hAut $(\bar{u})$ act on $\Omega^{\infty} M T \Theta$, we therefore have a zig-zag of maps

$$
\left(\Omega_{[[W, \lambda]]}^{\infty} M T \Theta\right) / / \operatorname{hAut}(\bar{u}) \longrightarrow \cdot \longleftarrow\left(\Omega_{\left[\left[W^{\prime}, \lambda^{\prime}\right]\right]}^{\infty} M T \Theta\right) / / \operatorname{hAut}(\bar{u})
$$

which induce isomorphisms on homology with coefficients in $A$. The argument is completed by the following lemma.

Lemma 2.1 The natural map $\mathrm{hAut}(\bar{u}) \rightarrow \operatorname{hAut}(u)$ is a weak equivalence. 
Proof Working in the categories of $\mathrm{GL}_{2 n}(\mathbb{R})$-spaces over $\Lambda$, or $\mathrm{GL}_{2 n+1}(\mathbb{R})$-spaces over $\Lambda$, we have

$$
\operatorname{map}_{\mathrm{GL}_{2 n}(\mathbb{R})}^{/ \Lambda}(\Theta, \Theta)=\operatorname{map}_{\mathrm{GL}_{2 n+1}(\mathbb{R})}^{/ \Lambda}\left(\mathrm{GL}_{2 n+1}(\mathbb{R}) \times_{\mathrm{GL}_{2 n}(\mathbb{R})} \Theta, \bar{\Theta}\right)
$$

but the natural $\mathrm{GL}_{2 n+1}(\mathbb{R})$-equivariant map $\mathrm{GL}_{2 n+1}(\mathbb{R}) \times_{\mathrm{GL}_{2 n}(\mathbb{R})} \Theta \rightarrow \bar{\Theta}$ has homotopy fiber $\mathrm{GL}_{2 n+1}(\mathbb{R}) / \mathrm{GL}_{2 n}(\mathbb{R}) \simeq S^{2 n}$ so is $2 n$-connected, whereas $\bar{u}: \bar{\Theta} \rightarrow \Lambda$ is $n$-coconnected, so the restriction map

$$
\operatorname{map}_{\mathrm{GL}_{2 n+1}(\mathbb{R})}^{/ \Lambda}(\bar{\Theta}, \bar{\Theta}) \longrightarrow \operatorname{map}_{\mathrm{GL}_{2 n+1}(\mathbb{R})}^{/ \Lambda}\left(\mathrm{GL}_{2 n+1}(\mathbb{R}) \times_{\mathrm{GL}_{2 n}(\mathbb{R})} \Theta, \bar{\Theta}\right)
$$

is an equivalence. The claim now follows by restricting to the path-components of homotopy equivalences.

Remark 2.2 This argument also gives a conclusion about homology with certain local coefficients. The maps (2.1) and (2.2) are in fact acyclic in a range of degrees [6, Corollary $1.9]$, and the maps $\psi^{q}$ are acyclic with $\mathbb{Z}\left[q^{-1}\right]$-module coefficients (as they are infinite loop maps which induce isomorphisms on homology with these coefficients) so remain so after taking homotopy orbits by hAut $(\bar{u})$.

So if $\mathcal{A}$ is a system of local coefficients on the middle space of the zig-zag (2.4), with typical fiber $A$ and having $v_{p}(\chi(W))=v_{p}\left(\chi\left(W^{\prime}\right)\right)$ for all primes $p$ which are not invertible in $\operatorname{End}_{\mathbb{Z}}(A)$, then there is also an isomorphism $H^{i}\left(\mathcal{M}^{\Lambda}(W, \lambda) ; \mathcal{A}\right) \cong H^{i}\left(\mathcal{M}^{\Lambda}\left(W^{\prime}, \lambda^{\prime}\right) ; \mathcal{A}\right)$ in a range of degrees.

\section{Proof of Theorems 1.3 and 1.4}

The proof of Theorem 1.3 is by an explicit construction of $\psi^{q}$ as a map of spectra. The main ingredient is a certain commutative diagram of spectra, which we first describe informally. It is

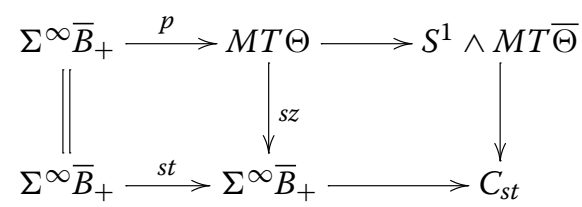

where $s: B \rightarrow \bar{B}$ is the natural map of Borel constructions. The map $s$ is homotopy equivalent to a smooth fiber bundle with fibers $S^{2 n}$ so we have a Becker-Gottlieb transfer $t: \Sigma^{\infty} \bar{B}_{+} \rightarrow \Sigma^{\infty} B_{+}$, factoring as a pre-transfer $p: \Sigma^{\infty} \bar{B}_{+} \rightarrow M T \Theta$ composed with a map $z: M T \Theta \rightarrow \Sigma^{\infty} B_{+}$induced by the zero section of $\theta$. The spectrum $C_{s t}$ is defined to be the homotopy cofiber of $s t$, and both rows are cofiber sequences. It follows that the right square in the diagram is a homotopy pullback, and hence we get the homotopy pullback diagram of infinite loop spaces (1.3) mentioned in the introduction. On spectrum homology the map st induces multiplication by $\chi\left(S^{2 n}\right)=2$, from which it follows that the homology and hence homotopy groups of $C_{s t}$ are 2-power torsion. The space $\bar{B}$ is path connected, because $W$ is, so $\pi_{0}\left(\Sigma^{\infty} \bar{B}_{+}\right)=H_{0}\left(\Sigma^{\infty} \bar{B}_{+} ; \mathbb{Z}\right)=\mathbb{Z}$. Thus $\pi_{0}\left(C_{s t}\right)=\mathbb{Z} / 2$, and the map $\Sigma^{\infty} \bar{B}_{+} \rightarrow C_{s t}$ is surjective on $\pi_{1}$ because st is injective on $\pi_{0}$.

To produce an endomorphism of $\Omega^{\infty} M T \Theta$ satisfying part (ii) of the theorem, it therefore suffices to produce an endomorphism of $\Sigma^{\infty} \bar{B}_{+}$over $C_{s t}$. For $q=1+2 k$, we may use the map id $+k s t: \Sigma^{\infty} \bar{B}_{+} \rightarrow \Sigma^{\infty} \bar{B}_{+}$which is obviously over $C_{s t}$, at least in the homotopy category, since $C_{s t}$ is the cofiber of the map st. In spectrum homology, st 
multiplies by $\chi\left(S^{2 n}\right)=2$ and hence id $+k s t$ induces multiplication by $1+2 k=q$ on $H_{*}\left(\Sigma^{\infty} \bar{B}_{+} ; \mathbb{Z}\right)$ ensuring part (iii) of the theorem. Furthermore, it acts by multiplication by $q$ on $\pi_{0} \Sigma^{\infty} \bar{B}_{+}=\pi_{0} Q\left(\bar{B}_{+}\right)=\mathbb{Z}$, so indeed sends $\Omega_{\chi}^{\infty} M T \Theta$ to $\Omega_{q \chi}^{\infty} M T \Theta$.

It remains to explain how to achieve part (i) of the theorem, that the continuous action of the topological monoid hAut $(\bar{\Theta})$ on the space $\Omega^{\infty} M T \Theta$ commutes with $\psi^{q}$. It is not sufficient that $\psi^{q}$ commutes up to homotopy with the action of individual elements of hAut $(\bar{\Theta})$, since we want to descend $\psi^{q}$ to the homotopy orbit space. To give a convincing proof, it seems best to spell out a point-set model for the square (1.3).

Proof of Theorem 1.3 As explained above, it remains to give a point-set model for the diagram (1.3) and the self-map id $+k s t$ of $Q\left(\bar{B}_{+}\right)$over $\Omega^{\infty} C_{s t}$, all of which commute strictly with the action of hAut $(\bar{\Theta})$.

We must adopt some conventions. Let us consider $\mathrm{GL}_{2 n}(\mathbb{R})$ as lying inside $\mathrm{GL}_{2 n+1}(\mathbb{R})$ using the last $2 n$ coordinates. Let us consider $\mathbb{R}^{N-1}$ as lying inside $\mathbb{R}^{N}$ as the subspace of vectors whose last coordinate is 0 , and take $\mathbb{R}^{\infty}$ to be the direct limit. To form the Borel constructions, we shall take $E \mathrm{GL}_{2 n}(\mathbb{R}):=\operatorname{Fr}_{2 n}\left(\mathbb{R}^{\infty}\right)$, and similarly take $E \mathrm{GL}_{2 n+1}(\mathbb{R}):=\operatorname{Fr}_{2 n+1}\left(\mathbb{R} \oplus \mathbb{R}^{\infty}\right)$. The map $\operatorname{Fr}_{2 n}\left(\mathbb{R}^{\infty}\right) \rightarrow \operatorname{Fr}_{2 n+1}\left(\mathbb{R} \oplus \mathbb{R}^{\infty}\right)$ which adds the basis vector of the first $\mathbb{R}$-summand as the first element of the $(2 n+1)$-frame is then equivariant for the inclusion $\mathrm{GL}_{2 n}(\mathbb{R}) \subset \mathrm{GL}_{2 n+1}(\mathbb{R})$.

Then we have $B \mathrm{GL}_{2 n+1}(\mathbb{R})=\mathrm{Gr}_{2 n+1}\left(\mathbb{R} \oplus \mathbb{R}^{\infty}\right)$, which we may filter in the usual way by $\mathrm{Gr}_{2 n+1}\left(\mathbb{R} \oplus \mathbb{R}^{N-1}\right)$. Pulling back this filtration along the map $\bar{\theta}: \bar{B} \rightarrow \mathrm{Gr}_{2 n+1}\left(\mathbb{R}^{\infty}\right)$, we set $\bar{B}_{N}:=(\bar{\theta})^{-1}\left(\operatorname{Gr}_{2 n+1}\left(\mathbb{R} \oplus \mathbb{R}^{N-1}\right)\right)$. There is an induced map $\bar{\theta}_{N}: \bar{B}_{N} \rightarrow \operatorname{Gr}_{2 n+1}\left(\mathbb{R} \oplus \mathbb{R}^{N-1}\right)$ and we shall write $\bar{\theta}_{N}^{*} \gamma^{\perp}=\bar{\theta}_{N}^{*} \gamma_{2 n+1, N}^{\perp}$ for the pullback of the $(N-2 n-1)$-dimensional bundle of orthogonal complements. Then $M T \bar{\Theta}$ is the spectrum with $N$ th space given by the Thom space $\left(\bar{B}_{N}\right)^{\bar{\theta}_{N}^{*} \gamma^{\perp}}$, so that

$$
\Omega^{\infty-1} M T \bar{\Theta}=\operatorname{colim}_{N \rightarrow \infty} \Omega^{N-1}\left(\bar{B}_{N}\right)^{\bar{\theta}_{N}^{*} \gamma^{\perp}} .
$$

We similarly define $\theta_{N}: B_{N} \rightarrow \operatorname{Gr}_{2 n}\left(\mathbb{R}^{N}\right)$, and hence the spectrum $M T \Theta$. There is a map

$$
\mathrm{Gr}_{2 n}\left(\mathbb{R}^{N-1}\right) \hookrightarrow \mathrm{Gr}_{2 n+1}\left(\mathbb{R} \oplus \mathbb{R}^{N-1}\right),
$$

given by direct sum with the 1-dimensional vector space given by the first $\mathbb{R}$-summand, which induces a map $B_{N-1} \rightarrow \bar{B}_{N}$. The map (3.1) is $2 n$-connected, but is covered by an $(N-2)$-connected map $\mathrm{Gr}_{2 n}\left(\mathbb{R}^{N-1}\right) \rightarrow S\left(\gamma_{2 n+1, N}\right)$ and hence gives a $(N-2)$-connected $\operatorname{map} B_{N-1} \rightarrow S\left(\bar{\theta}_{N}^{*} \gamma_{2 n+1, N}\right)$. Passing to Thom spaces, this gives a $(2 N-2 n-2)$-connected map

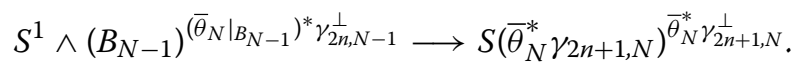

These combine to define a map from $M T \Theta$ to the spectrum whose $(N-1)$ st space is $S\left(\bar{\theta}_{N}^{*} \gamma_{2 n+1, N}\right)^{\bar{\theta}_{N}^{*}} \gamma_{2 n+1, N}^{\perp}$, and this map is a weak equivalence. This map is also hAut $(\bar{\Theta})$ equivariant. (This weak equivalence does not come with a spectrum map in the other direction, let alone an equivariant one.)

The square (1.3) will be assembled from a square of spaces fibered over $\bar{B}_{N}$, and we first explain the constructions on fibers. Let $V \in \mathrm{Gr}_{2 n+1}\left(\mathbb{R}^{N}\right)$ and write $S(V)$ for the unit sphere of $V$ and $S^{V}$ for the one-point compactification. If $x \in \mathbb{R}^{N}$, we shall write $\pi_{V}(x) \in V$ for 
the orthogonal projection. If $x \in V \backslash 0$, we shall write $\pi_{S}(x)=x /|x| \in S(V)$ for the nearest point in the sphere. We will describe certain explicit maps $p(V): S^{V} \rightarrow S\left(V \varepsilon^{\varepsilon^{1}}\right.$ and $z(V): S(V)^{\varepsilon^{1}} \rightarrow S(V)_{+} \wedge S^{V}$, and explain how the composition $z(V) \circ p(V)$ gives rise to a model for the Becker-Gottlieb transfer for a linear sphere bundle. (Indeed, we will just unwrap the definition of $[1$, Sect. 3$]$ in this case.)

The map

$$
p(V): S^{V} \longrightarrow S(V)^{\varepsilon^{1}}
$$

is induced by the Pontryagin-Thom construction applied to the embedding $S(V) \subset V$. In formulas, we can take, e.g.

$$
p(V)(x)=\left(\pi_{S}(x), \log |x|\right) \in S(V)_{+} \wedge S^{1}=S(V)^{\varepsilon^{1}}
$$

when $x \neq 0, \infty \in S^{V}$. The Thom space $S(V)^{\varepsilon^{1}}$ is homeomorphic to the quotient $S^{V} / S^{0}$, and under this identification, the map $p(V)$ is the quotient map.

The map

$$
z(V): S(V)^{\varepsilon^{1}} \longrightarrow S(V)^{T S(V) \oplus \varepsilon^{1}}=S(V)_{+} \wedge S^{V}
$$

is given by the zero section of the tangent bundle of $S(V)$. In formulas, it sends $(x, t) \in$ $S(V) \times \mathbb{R} \subset S(V)^{\varepsilon^{1}}$ to $(x, t x) \in S(V) \times V \subset S(V)_{+} \wedge S^{V}$.

If we compose these two maps and smash with $S^{V^{\perp}}$, we get

$$
S^{N}=S^{V} \wedge S^{V^{\perp}} \stackrel{p(V) \wedge \text { id }}{\longrightarrow} S(V)^{\varepsilon^{1}} \wedge S^{V^{\perp}} \stackrel{z(V) \wedge \text { id }}{\longrightarrow} S(V)_{+} \wedge S^{V} \wedge S^{V^{\perp}}=S(V)_{+} \wedge S^{N} .
$$

Finally, we write $s(V): S(V)_{+} \wedge S^{N} \rightarrow S^{N}$ for the map induced by collapsing $S(V)$ to a point. Then the composition

$$
b(V)=s(V) \circ(z(V) \wedge \mathrm{id}) \circ(p(V) \wedge \mathrm{id}): S^{N} \longrightarrow S^{N}
$$

is a continuous map of degree $\chi\left(S^{2 n}\right)=2$ (by the Poincaré-Hopf theorem, see [1, Theorem 2.4]), depending continuously on the point $V \in \mathrm{Gr}_{2 n+1}\left(\mathbb{R}^{N}\right)$. The resulting continuous map $b: G_{2 n+1}\left(\mathbb{R}^{N}\right) \rightarrow \Omega^{N} S^{N}$ in the limit gives a map $B G L_{2 n+1}(\mathbb{R}) \rightarrow Q S^{0}$ which is a model for the Becker-Gottlieb transfer of the sphere bundle over the space $B \mathrm{GL}_{2 n+1}(\mathbb{R}) \simeq$ $B \mathrm{O}(2 n+1)$.

Now consider the diagram

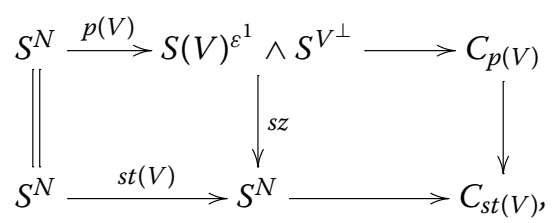

where the entries in the right column are the mapping cylinders. Since $p(V)$ induces a homeomorphism $S^{N} / S^{V^{\perp}} \rightarrow S(V)^{\varepsilon} \wedge S^{V^{\perp}}$, it follows from the Puppe sequence that there is a canonical induced homeomorphism $C_{p(V)} \cong S^{1} \wedge S^{V^{\perp}}$. Since $s t(V)$ has degree 2, there is a homotopy equivalence from $C_{s t(V)}$ to a $\bmod 2$ Moore space, but this is not quite 
sufficiently canonical for our purposes (since we get a different mod 2 Moore space for each $V$ ). We have proved that for each $V \in \mathrm{Gr}_{2 n+1}\left(\mathbb{R}^{N}\right)$ there is a canonical commutative diagram

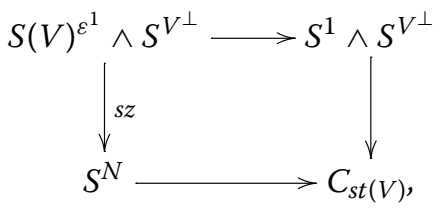

which is a pushout and homotopy pushout.

There is a canonical homotopy from the composition of $s t(V): S^{N} \rightarrow S^{N}$ and $S^{N} \rightarrow$ $C_{s t(V)}$ to the constant map. Suspending once, $S^{1} \wedge S^{N} \rightarrow S^{1} \wedge S^{N} \rightarrow S^{1} \wedge C_{s t(V)}$ is canonically null homotopic. If $k \geq 0$ is an integer, we may use the $S^{1}$ coordinate to form the sum of the identity map $1: S^{1} \wedge S^{N} \rightarrow S^{1} \wedge S^{N}$ and $k$ copies of the map $s t(V): S^{1} \wedge S^{N} \rightarrow S^{1} \wedge S^{N}$. We obtain a diagram

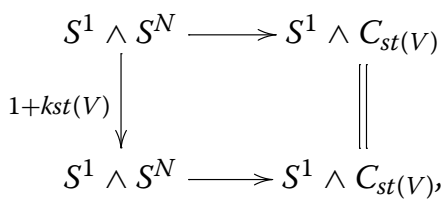

which commutes up to a canonical homotopy. (The canonical nullhomotopy of each $s t$ gives a homotopy from $1+k s t$ to the sum of the identity map and $k$ copies of the constant map; this is in turn canonically homotopic to the identity map.) The homotopy class of the map $1+k s t(V): S^{N} \rightarrow S^{N}$ is determined by its degree which is $2 k+1$, but the actual map depends in a non-trivial way on $V \in \mathrm{Gr}_{2 n+1}\left(\mathbb{R}^{N}\right)$.

All spaces in the diagram "vary continuously in $V$," in the sense that they are fibers over $V$ of fiber bundles over $\mathrm{Gr}_{2 n+1}\left(\mathbb{R}^{N}\right)$. The commutative diagram (3.2) in the category of spaces over $\operatorname{Gr}_{2 n+1}\left(\mathbb{R}^{N}\right)$ may be pulled back along $\bar{\theta}_{N}: \bar{B}_{N} \rightarrow \mathrm{Gr}_{2 n+1}\left(\mathbb{R}^{N}\right)$ to form a diagram

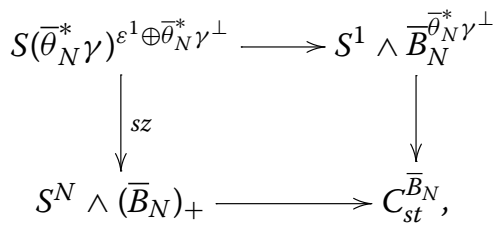

which is again a pushout and homotopy pushout, where $C_{s t}^{\bar{B}_{N}}$ is the mapping cylinder of the map $S^{N} \wedge\left(\bar{B}_{N}\right)_{+} \rightarrow S^{N} \wedge\left(\bar{B}_{N}\right)_{+}$given on $(v, x) \in S^{N} \times \bar{B}_{N}$ by $s t(v, x)=(s t(f(x)) v, x)$.

Similarly, the diagrams (3.3) assemble over $V$ to a diagram

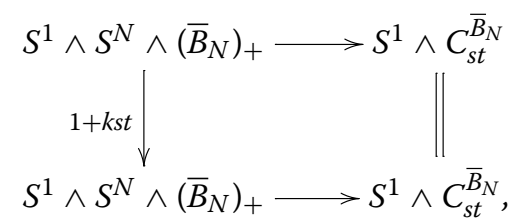

which commutes up to a canonical homotopy. 
Applying $\Omega^{N+1} S^{1} \wedge(-)$ to the diagram (3.4) and letting $N \rightarrow \infty$, we get a model for (1.3). The monoid hAut $(\bar{\Theta})$ acts on the whole diagram (3.4), since it acts on $\bar{B}_{N}$ over $\mathrm{Gr}_{2 n+1}\left(\mathbb{R}^{N}\right)$. This gives a weak equivalence from $\Omega^{\infty} M T \Theta$ to the homotopy pullback in (1.3), which is also an hAut $(\bar{\Theta})$ equivariant map. The monoid hAut $(\bar{\Theta})$ also acts on the diagram (3.5), including the homotopy, and after applying $\Omega^{N+1}$ and taking $N \rightarrow \infty$, we obtain a self-map of $Q\left(\bar{B}_{+}\right)$which is over $\Omega^{\infty} C_{s t}$ up to a specified homotopy. Again this self-map and the specified homotopy commute strictly with the action of hAut $(\bar{\Theta})$ since both the map and the homotopy arose from fiberwise constructions over $\mathrm{Gr}_{2 n+1}\left(\mathbb{R}^{N}\right)$.

Finally, the self-map of $Q\left(\bar{B}_{+}\right)$induces an hAut $(\bar{\Theta})$-equivariant self-map of the homotopy pullback of $Q\left(\bar{B}_{+}\right) \rightarrow \Omega^{\infty} C_{s t} \leftarrow \Omega^{\infty-1} M T \bar{\Theta}$, and we have seen that this pullback is weakly equivalent to $\Omega^{\infty} M T \Theta$ by an hAut $(\bar{\Theta})$-equivariant map.

Proof of Theorem 1.4 We continue with the notation developed above. The spectrum homology of $C_{s t}$ is all 2-torsion, so the localization $C_{s t}\left[\frac{1}{2}\right]$ as a spectrum is contractible. However, the localized space $\left(\Omega^{\infty} C_{s t}\right)\left[\frac{1}{2}\right]$ is not contractible since it has two components. Instead, there is a spectrum map $w_{2 n}: C_{s t} \rightarrow H \mathbb{F}_{2}$ which becomes an isomorphism in homology of infinite loop spaces with coefficients in any $\mathbb{Z}\left[\frac{1}{2}\right]$-module. Similarly, the map

$$
\Omega^{\infty} M T \Theta \longrightarrow Q\left(\bar{B}_{+}\right) \times_{\Omega^{\infty} H \mathbb{F}_{2}} \Omega^{\infty-1} M T \bar{\Theta}
$$

induces an isomorphism in homology with coefficients in any $\mathbb{Z}\left[\frac{1}{2}\right]$-module, and hence a weak equivalence of localized spaces. The spectrum map $2: S^{0} \rightarrow S^{0}$ induces a self-map of $Q\left(\bar{B}_{+}\right)$commuting with the action of $\mathrm{hAut}(\bar{\Theta})$ and whose restriction to the even-degree path components commutes with the map to $\Omega^{\infty} H \mathbb{F}_{2}$. This self-map can be used in place of $1+k s t$ to produce $\psi^{2}$.

\section{An example}

In this section, we will give an example to show that in Theorem 1.1 it is indeed necessary to take homology with certain primes inverted. We will take as an example the 6-manifolds $V_{d}$ given by a smooth degree $d$ hypersurface in $\mathbb{C P}^{4}$, which we have studied in detail in [8, Sect. 5.3]. Any unattributed claims about these manifolds may be found there. We will also consider their stabilizations

$$
V_{d, g}:=V_{d} \# g\left(S^{3} \times S^{3}\right)
$$

obtained by connect-sum of $V_{d}$ with $g$ copies of $S^{3} \times S^{3}$, which contain

$$
g\left(V_{d, g}\right)=g+\frac{1}{2}\left(d^{4}-5 d^{3}+10 d^{2}-10 d+4\right)
$$

copies of $S^{3} \times S^{3}$.

Theorem 4.1 Let $p \geq 7$ be a prime number, and suppose that $g\left(V_{d, g}\right) \geq 9$. Then

$$
H^{3}\left(\mathcal{M}^{\text {or }}\left(V_{d, g}\right) ; \mathbb{Z}_{(p)}\right) \cong \mathbb{Z}_{(p)} / \operatorname{gcd}(d, g) .
$$

The formula $\chi=\chi\left(V_{d, g}\right)=d\left(10-10 d+5 d^{2}-d^{3}\right)-2 g$ implies that $\operatorname{gcd}(d, g)=\operatorname{gcd}(d, \chi)$, so the theorem may also be written

$$
H^{3}\left(\mathcal{M}^{\text {or }}\left(V_{d, g}\right) ; \mathbb{Z}_{(p)}\right) \cong \mathbb{Z} / p^{\min \left(v_{p}(d), v_{p}(\chi)\right)} \mathbb{Z}
$$


Hence the moduli spaces for the oriented stably diffeomorphic manifolds $V_{d, g}$ and $V_{d, g^{\prime}}$ have isomorphic $H^{3}\left(-; \mathbb{Z}_{(p)}\right)$ if and only if $v_{p}\left(\chi\left(V_{d, g}\right)\right)=v_{p}\left(\chi\left(V_{d, g^{\prime}}\right)\right)$, provided those $p$-adic valuations are at most $v_{p}(d)$.

Proof of Theorem 4.1 In [8, Sect. 5.3], we computed the $\mathbb{Q}$-cohomology of $\mathcal{M}^{\text {or }}\left(V_{d, g}\right)$ in a stable range. We will refer to details of the notation from that discussion, which differs slightly from the notation used earlier in this note.

Firstly, the $\mathbb{Q}$-cohomology calculation goes through without significant changes for $\mathcal{M}^{\text {or }}\left(V_{d, g}\right)$, because $V_{d, g}$ and $V_{d}$ have the same Moore-Postnikov 3-stage, and because any orientation preserving diffeomorphism of $V_{d, g}$ must also act trivially on $H^{2}\left(V_{d, g} ; \mathbb{Z}\right)$. The only difference is that the formula for the $d_{3}$-differential now involves characteristic numbers of $V_{d, g}$, which can be calculated to give

$$
\begin{aligned}
d_{3}\left(\kappa_{p_{2}}\right) & =0 \\
d_{3}\left(\kappa_{p_{1}^{2}}\right) & =0 \\
d_{3}\left(\kappa_{t e}\right) & =\kappa_{e}=\chi\left(V_{d, g}\right)=d\left(10-10 d+5 d^{2}-d^{3}\right)-2 g, \\
d_{3}\left(\kappa_{t^{2} p_{1}}\right) & =2 \kappa_{t p_{1}}=2 d\left(5-d^{2}\right), \\
d_{3}\left(\kappa_{t^{4}}\right) & =4 \kappa_{t_{3}}=4 d .
\end{aligned}
$$

Secondly, the $\mathbb{Q}$-cohomology calculation yields an analogous $\mathbb{Z}_{(p)}$-cohomology calculation for large enough primes $p$. Specifically the spectrum $M T \theta_{d}$ is (-6)-connected, so by the Atiyah-Hirzebruch spectral sequence the Hurewicz map

$$
\pi_{i}\left(M T \theta_{d}\right)_{(p)} \longrightarrow H_{i}\left(M T \theta_{d} ; \mathbb{Z}_{(p)}\right) \cong H_{i+6}\left(B_{d} ; \mathbb{Z}_{(p)}\right)
$$

is an isomorphism as long as $i<2 p-3-6$, so as long as $i \leq 5$ since we have assumed that $p \geq 7$. As $p$ is odd we have

$$
H^{*}\left(B_{d} ; \mathbb{Z}_{(p)}\right)=H^{*}\left(B S O(6) \times K(\mathbb{Z}, 2) ; \mathbb{Z}_{(p)}\right)=\mathbb{Z}_{(p)}\left[p_{1}, p_{2}, e, t\right] .
$$

Thus we have $\pi_{1}\left(\Omega_{0}^{\infty} M T \theta_{d}\right)_{(p)}=0, \pi_{2}\left(\Omega_{0}^{\infty} M T \theta_{d}\right)_{(p)} \cong \mathbb{Z}_{(p)}^{5}$ with the isomorphism given by the tautological classes $\kappa_{p_{2}}, \kappa_{p_{1}^{2}}, \kappa_{t e}, \kappa_{t^{2} p_{1}}, \kappa_{t^{4}}$, and $\pi_{3}\left(\Omega_{0}^{\infty} M T \theta_{d}\right)_{(p)}=0$. Therefore

$$
H^{i}\left(\mathcal{M}^{\theta_{d}}\left(V_{d, g}, \ell_{V_{d, g}}\right) ; \mathbb{Z}_{(p)}\right)= \begin{cases}\mathbb{Z}_{(p)} & i=0 \\ 0 & i=1 \\ \mathbb{Z}_{(p)}\left\{\kappa_{p_{2}}, \kappa_{p_{1}^{2}}, \kappa_{t e}, \kappa_{t^{2} p_{1}}, \kappa_{t^{4}}\right\} & i=2 \\ 0 & i=3 .\end{cases}
$$

The submonoid $G \leq \operatorname{hAut}(u)$ of those path components which stabilize $\left[V_{d, g}, \ell_{V_{d, g}}\right]$ is path connected, and as the map $u: B_{d} \rightarrow B S O(6) \times K(\mathbb{Z}, 2)$ is a $\mathbb{Z}_{(p)}$-homology equivalence, since $p$ is odd, we also have that $\pi_{i}(G) \otimes \mathbb{Z}_{(p)}=0$ for $i>0$. Thus the map $\mathcal{M}^{\theta_{d}}\left(V_{d, g}, \ell_{V_{d, g}}\right) \rightarrow \mathcal{M}^{\mu}\left(V_{d, g}, u \circ \ell_{V_{d, g}}\right)$ is a $\mathbb{Z}_{(p)}$-homology equivalence.

It remains to study the Serre spectral sequence for the fibration sequence

$$
\mathcal{M}^{\mu}\left(V_{d, g}, u \circ \ell_{V_{d, g}}\right) \longrightarrow \mathcal{M}^{\text {or }}\left(V_{d, g}\right) \longrightarrow K(\mathbb{Z}, 3),
$$

which in low degrees has a single differential

$$
d_{3}: E_{3}^{0,2}=\mathbb{Z}_{(p)}\left\{\kappa_{p_{2}}, \kappa_{p_{1}^{2}}, \kappa_{t e}, \kappa_{t^{2} p_{1}}, \kappa_{t^{4}}\right\} \longrightarrow E_{3}^{3,0}=H^{3}\left(K(\mathbb{Z}, 3) ; \mathbb{Z}_{(p)}\right)=\mathbb{Z}_{(p)}
$$


given by the formula above, so $H^{3}\left(\mathcal{M}^{\text {or }}\left(V_{d, g}\right) ; \mathbb{Z}_{(p)}\right)$ is given by the cokernel of this differential. The claim now follows by the identity of ideals

$$
\left(4 d, 2 d\left(5-d^{2}\right), d\left(10-10 d+5 d^{2}-d^{3}\right)-2 g\right)=(d, g)
$$

of $\mathbb{Z}_{(p)}$, using again that $p$ is odd.

\section{Acknowledgements}

SG was supported by the European Research Council (ERC) under the European Union's Horizon 2020 research and innovation programme (Grant Agreement No. 682922), the Danish National Research Foundation through the Centre for Symmetry and Deformation (DNRF92), and the EliteForsk Prize. ORW was supported by EPSRC grant EP/M027783/1, the ERC under the European Union's Horizon 2020 research and innovation programme (Grant Agreement No. 756444), and a Philip Leverhulme Prize from the Leverhulme Trust.

\section{Author details}

'Department of Mathematics, University of Copenhagen, Copenhagen, Denmark, ${ }^{2}$ Centre for Mathematical Sciences, Wilberforce Road, Cambridge CB3 OWB, UK.

Received: 21 December 2019 Accepted: 23 March 2020 Published online: 17 April 2020

\section{References}

1. Becker, J.C., Gottlieb, D.H.: The transfer map and fiber bundles. Topology 14, 1-12 (1975)

2. Bendersky, M., Miller, J.: Localization and homological stability of configuration spaces. Q. J. Math. 65(3), 807-815 (2014)

3. Cantero, F., Palmer, M.: On homological stability for configuration spaces on closed background manifolds. Doc. Math. 20, 753-805 (2015)

4. Friedrich, N.: Homological stability of automorphism groups of quadratic modules and manifolds. Doc. Math. 22 1729-1774 (2017)

5. Galatius, S., Randal-Williams, O.: Stable moduli spaces of high-dimensional manifolds. Acta Math. 212(2), 257-377 (2014)

6. Galatius, S., Randal-Williams, O.: Homological stability for moduli spaces of high dimensional manifolds. II. Ann. Math. (2) 186(1), 127-204 (2017)

7. Galatius, S., Randal-Williams, O.: Homological stability for moduli spaces of high dimensional manifolds. I. J. Am. Math. Soc. 31(1), 215-264 (2018)

8. Galatius, S., Randal-Williams, O.: Moduli spaces of manifolds: a user's guide. In: Handbook of Homotopy Theory, pp. 445-487. Chapman \& Hall/CRC, CRC Press, Boca Raton, FL (2019)

9. Harer, J.L.: Stability of the homology of the mapping class groups of orientable surfaces. Ann. Math. (2) $\mathbf{1 2 1}(2)$ 215-249 (1985)

10. Krannich, M.: On characteristic classes of exotic manifold bundles, Mathematische Annalen (2019), arXiv:1802.02609

11. Madsen, l., Tillmann, U.: The stable mapping class group and $Q\left(\mathbb{C P}_{+}^{\infty}\right)$. Invent. Math. 145(3), 509-544 (2001)

12. Madsen, l., Weiss, M.: The stable moduli space of Riemann surfaces: Mumford's conjecture. Ann. Math. (2) 165(3), 843-941 (2007)

13. Tillmann, U.: On the homotopy of the stable mapping class group. Invent. Math. 130(2), 257-275 (1997)

\section{Publisher's Note}

Springer Nature remains neutral with regard to jurisdictional claims in published maps and institutional affiliations. 